\section{Pleuramesotheliom: Prophylaktische Bestrahlung}

Große thorakale Eingriffe können beim Pleuramesotheliom dazu führen, dass Metastasen entstehen. Ist es deshalb angezeigt, sicherheitshalber schon mal zu bestrahlen, auch wenn noch keine Tochtergeschwulste zu erkennen sind?

$\mathrm{D}$ er Einsatz der prophylaktischen Radiotherapie beim malignen Pleuramesotheliom bleibt kontrovers. Sie dient vor allem zur Vorbeugung von behandlungsbedingten Metastasen (sogenannte "procedure-tract metastases", PTM). In vielen Zentren wird aber erst nach Entwicklung von PTM bestrahlt. Nun wurde in einer multizentrischen britischen Phase-III-Studie der Nutzen einer prophylaktischen mit dem einer verzögerten Radiotherapie verglichen.

203 Patienten, die sich in den 35 Tagen vor der Rekrutierung einer umfassenden Intervention am Rippenfell unterzogen hatten (z.B. offene Pleurabiopsie, operative Thorakotomie) erhielten randomisiert entweder eine sofortige Radiotherapie (21 Gy in 3 Fraktionen) innerhalb von 42 Tagen nach der Intervention oder eine verzögerte Radiotherapie (21 Gy in 3 Fraktionen) nach Diagnose von PTM. Stratifiziert wurde nach histologischem Subtyp und der Art des Eingriffs. Primärer Endpunkt war die Inzidenz von PTM binnen 12 Monaten innerhalb von $7 \mathrm{~cm}$ um den Ort der Intervention.

Es zeigte sich kein signifikanter Unterschied in der PTM-Inzidenz zwischen der Gruppe mit sofortiger versus verzö- gerter Radiotherapie ( 9 vs. $16 \%$; Hazard Ratio 0,$51 ; p=0,14)$. Eine schwerwiegende Nebenwirkung bei einem Patienten mit sofortiger Radiotherapie waren schmerzhafte PTM im Bestrahlungsfeld. Häufige Nebenwirkungen der sofortigen Radiotherapie betrafen die Haut. Die sofortige Radiotherapie war nicht kosteneffektiv.

Fazit: Diesen Daten zufolge ist es nicht gerechtfertigt, alle Patienten mit Mesotheliom nach einer großen thorakalen Intervention prophylaktisch zu bestrahlen, um behandlungsbedingten Metastasen vorzubeugen.

Christina Berndt

O'Clive A et al. Prophylactic radiotherapy for the prevention of procedure-tract metastases after surgical and large-bore pleural procedures in malignant pleural mesothelioma (SMART): a multicentre, open-label, phase 3, randomised controlled trial. Lancet Oncol. 2016;17(8):1094-104.

\section{Atemnot durch fortgeschrittenes NSCLC oder Mesotheliom: Akupunktur und/oder Morphin?}

\section{Zur Linderung von Atemnot bei Tumorpatienten werden häufig Opioide ge- geben, obschon sie dafür nicht zugelassen sind. Ob auch die Akupunktur bei Atemnot hilfreich sein kann, wurde nun in einer Phase-II-Studie untersucht.}

\begin{abstract}
$A_{t}^{u+n}$ ufgenommen wurden 173 ambulante Patienten mit nichtkleinzelligem Lungenkarzinom (NSCLC) oder Mesotheliom, die unter Atemnot litten. In Ruhe musste ihr Dyspnoe-Score $\geq 4$ auf einer visuellen Analogskala (VAS) betragen. Um die Wirksamkeit der Akupunktur zu prüfen, erhielten die Patien-
\end{abstract}

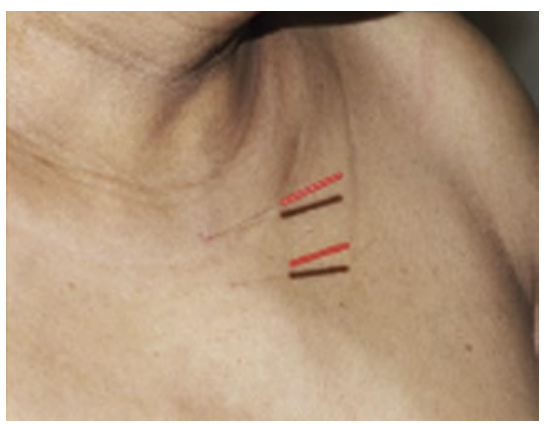

Abb. 1: Unter anderem am Sternum wurden die Patienten akupunktiert. ten entweder diese alleine (Arm A), Morphin alleine (Arm M) oder beides (Arm AM). Die Akupunktur wurde am oberen Sternum (Abb. 1), thorakal paravertebral, an den Trapezius-Triggerpunkten und an Punkt LI4 nahe dem Daumengrundgelenk durchgeführt. Für ein längeres Anhalten der Wirkung wurden zusätzlich am Manubrium sterni semipermanente Akupunkturnadeln gesetzt, die bei verstärkter Atemnot vom Patienten selbst massiert werden konnten. Primärer Endpunkt war der Anteil der Patienten, der nach 4 Stunden eine Verbesserung um $\geq 1,5$ Punkte auf der VAS zeigte.

Eine solche Verbesserung ergab sich bei $74 \%, 60 \%$ bzw. $66 \%$ der Patienten in den Studienarmen A, M und AM, bei $45 \%$ der Teilnehmer auch noch nach 2 Wochen. Es gab keine signifikanten Unterschiede zwischen den Studienarmen, auch nicht in den Lungenfunktionstests.
Die VAS-Werte für Entspannung verbesserten sich in Arm A um 1,06 Punkte sowie in Arm AM um 1,48 Punkte (Arm M -0,19 Punkte; $\mathrm{p}<0,001$ ). An Tag 7 verbesserte sich außerdem der Angst-Score in Arm A um 1,5 und in Arm AM um 1,2 Punkte, während er sich im Arm $M$ nicht veränderte $(p=0,003)$. In Arm A erhielten weniger Patienten $\geq 1$ Morphindosis verglichen mit Arm M oder AM (21 vs. 87 vs. $87 \%$; $\mathrm{p}<0,001)$. Die Möglichkeit, die Akupunkturwirkung durch Massieren der Verweil-Akupunkturnadeln zu verstärken, wurde in den entsprechenden Gruppen häufig genutzt (bis zu $32 \mathrm{mal}$ pro Tag).

Fazit: Akupunktur, Morphin oder die Kombination aus beiden konnten die Atemnot bei Patienten mit NSCLC oder Mesotheliom signifikant lindern. Die Akupunktur wirkte Angst mindernd und verringerte den Morphingebrauch. Sie stellt daher eine Alternative zum Einsatz von Opioiden dar. Brigitte Schalhorn

Minchom A et al. A randomised study comparing the effectiveness of acupuncture or morphine versus the combination for the relief of dyspnoea in patients with advanced non-small cell lung cancer and mesothelioma. Eur J Cancer. 2016;61:102-10. 